\title{
Intravascular lithotripsy with peripheral Shockwave catheter - a breakthrough in calcified carotid artery stenosis treatment
}

\author{
Marcin Misztal ${ }^{1,2}$, Mariusz Trystuła $^{1}$, Małgorzata Konieczyńska ${ }^{3}$, Robert Musiał ${ }^{4}$, Jacek Legutko², \\ Piotr Pieniążek ${ }^{1,2}$ \\ ${ }^{1}$ Vascular Surgery and Endovascular Procedures Department, John Paul II Hospital, Krakow, Poland \\ ${ }^{2}$ Department of Interventional Cardiology, Jagiellonian University Medical College, John Paul II Hospital, Krakow, Poland \\ ${ }^{3}$ Department of Quick Diagnosis, John Paul II Hospital, Krakow, Poland \\ ${ }^{4}$ Third Department of Anesthesiology, John Paul II Hospital, Krakow, Poland
}

Adv Interv Cardiol 2020; 16, 4 (62): 491-494

DOI: https://doi.org/10.5114/aic.2020.101778

Vascular calcium alters wall physical properties and consequently calcified vessels negatively affect stent expansion and apposition. Heavily calcified lesions may be difficult to dilate properly during an interventional procedure and thus are associated with a lower rate of technical success and a higher rate of complications. There are two main forms of calcification described in the peripheral vasculature: intimal ("coral reef"), which can protrude into the lumen, and medial (Mönckeberg's sclerosis), which impairs wall compliance and elasticity [1]. It has been proved that calcium acts as a physical barrier to anti-proliferative drug penetration and distribution [2]. Appropriate deployment of the stent, good stent apposition and proper coverage of the target lesion sometimes seem to be extremely challenging due to calcium deposits. Our state-of-the-art instrumentarium incorporates new techniques to treat calcified lesions: specialty balloons (e.g. cutting, scoring, ultra-high-pressure), rotablation and atherectomy. We must remember that "debulking" devices may have a limited effect on deep calcium deposits [3].

This new concept of intravascular lithotripsy (IVL) was transferred from kidney stone treatment used for several decades. Intravascular lithotripsy uses high-speed pulsatile sonic pressure waves that pass through soft tissues and interact strongly with calcium.

The technology gained the European CE Mark in May 2017 for coronary arteries. Then in June 2017 IVL gained the CE Mark and FDA approval for treatment of calcified lesions in lower limb atherosclerosis. Clinical data confirming safety and effectiveness of the IVL method can be found in the literature. One of the first published trials was the multicenter, prospective study Disrupt CAD, where the efficacy of lithoplasty in a group of 60 patients with severely calcified coronary artery lesions was investigated [4]. A subsequent study was Disrupt PAD II, which enrolled 60 subjects with complex, calcified peripheral stenosis treated with the IVL technique and followed for 12 months [5]. By contrast, we still lack studies enrolling patients with highly calcified plaques in carotid or innominate arteries. In the literature we can only find a few reports presenting single cases of carotid stenting assisted with intravascular lithotripsy. All cases reported were performed with coronary $\mathrm{C}^{2} 4.0 / 12 \mathrm{~mm}$ catheters $[6,7]$.

We present the first-in-man use of a peripheral lithotripsy catheter (Shockwave Medical Inc, Fremont, California) during a procedure of carotid artery stenting. The Bioethics Committee at the Regional Medical Chamber in Krakow, Poland approved the project of IVL usage in carotid arteries (opinion number: 178/KB/OIL/2019 dated 09.07.2019). An 82-year-old man was admitted to our hospital after left hemisphere ischemic stroke manifested in September 2020. Cerebral computed tomography (CT) scan showed a hypodense lesion in his left frontoparietal cortex. Carotid Doppler duplex imaging revealed extremely calcified tight stenosis of both internal carotid arteries. In compliance with our Vascular Team decision the patient was scheduled for carotid artery endarterectomy (CEA). Fortunately for the patient we decided to perform dedicated CT-angio with detailed assessment of the anatomy of vasculature and morphology of atherothrombotic plaques. Due to the very extensive calcific plaque

\section{Corresponding author:}

Marcin Misztal PhD, Vascular Surgery and Endovascular Procedures Department, John Paul II Hospital, 80 Prądnicka St, $31-302$ Krakow, Poland, phone: +48 606265 579, e-mail: marcin.misztal1@gmail.com

Received: 20.11.2020, accepted: 21.11.2020. 

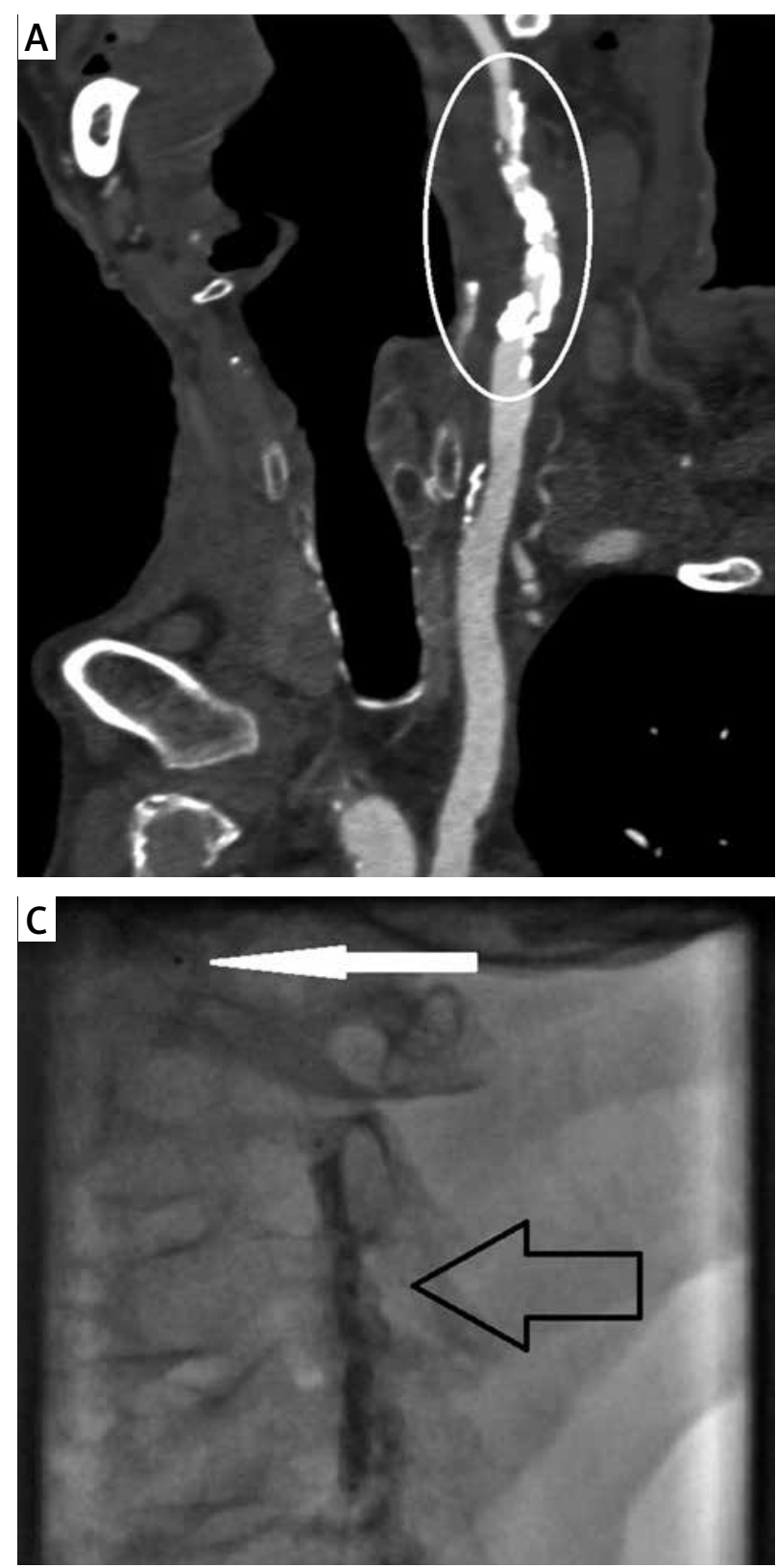

our patient was disqualified from surgery (Figure 1 A). The rationale for carotid artery stenting with off-label use of IVL was precisely explained to the patient and informed consent was obtained. Standard medical therapy was prescribed and clopidogrel in a loading dose of $300 \mathrm{mg}$ was given 2 days before the procedure. Briefly, under local anesthesia the right femoral artery was cannulated and selective angiography of the left internal carotid artery was performed (Figure $1 \mathrm{~B}$ ). Patient ECG was monitored for the whole procedure, Quick-Combo electrodes (Physio-Control, LIFEPAK) were implemented to protect the patient from prolonged bradycardia or asystole. Due to visible plaque eccentricity and extreme calcifications we had difficulties with crossing the lesion and distal filter positioning. After embolic protection system

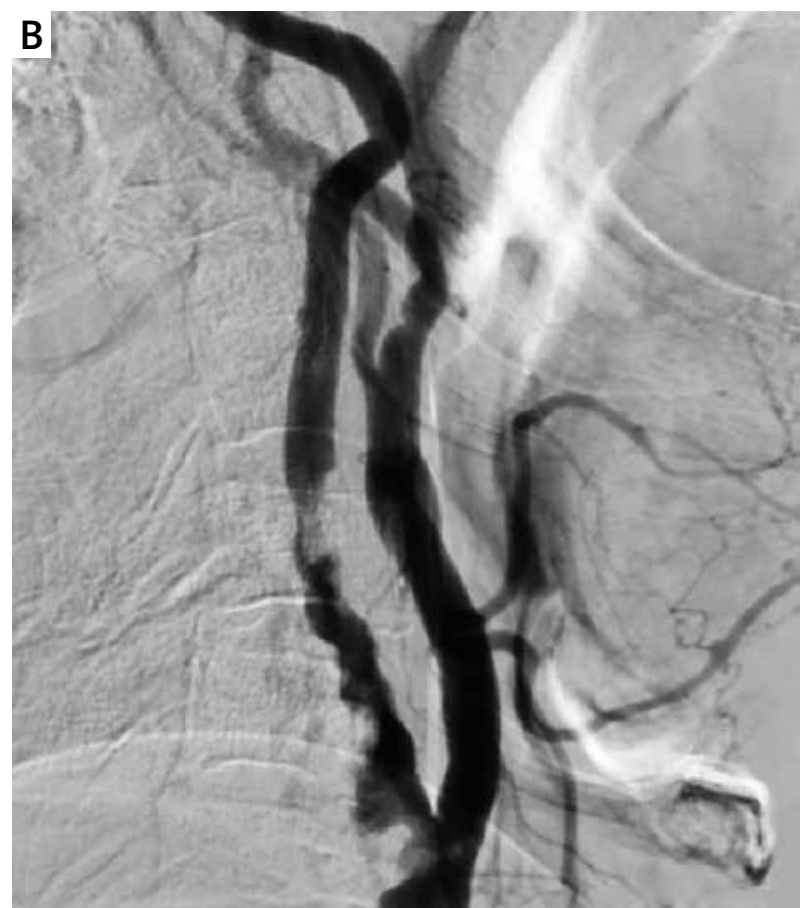

Figure 1. A-CT scan of severe calcification in left internal carotid artery (LICA). B - Digital subtraction angiography of left carotid arteries. C - Lithotripsy balloon inflation - black arrow (Spider FX neuroprotection system - white arrow)

deployment (SpiderFX, Medtronic, US) a Shockwave S4 4.0/40 $\mathrm{mm}$ over-the-wire peripheral lithoplasty balloon (Shockwave Medical, Fremont, California) was applied at the lesion level. It was inflated once at 2 atm; 20 pulses of energy were applied. We continued with 3 consecutive attempts of $4 \mathrm{~atm}$ inflation and 20 pulses of ultrasound energy lasting $20 \mathrm{~s}$ and final inflation to $6 \mathrm{~atm}$ without energy application (Figure $1 \mathrm{C}$ ). We should note that the patient showed ideal tolerance of the lithoplasty device. We did not observe any hemodynamic reaction, and heart rate and pressure were stable during IVL maneuvers. Once the lithoplasty predilation was completed, a 7.0/40 mm self expanding carotid Wallstent (BSC, US) was deployed and postdilated with two sequential $12 \mathrm{~atm}$ inflations of a 5.0/20 mm Sterling balloon (BSC, 

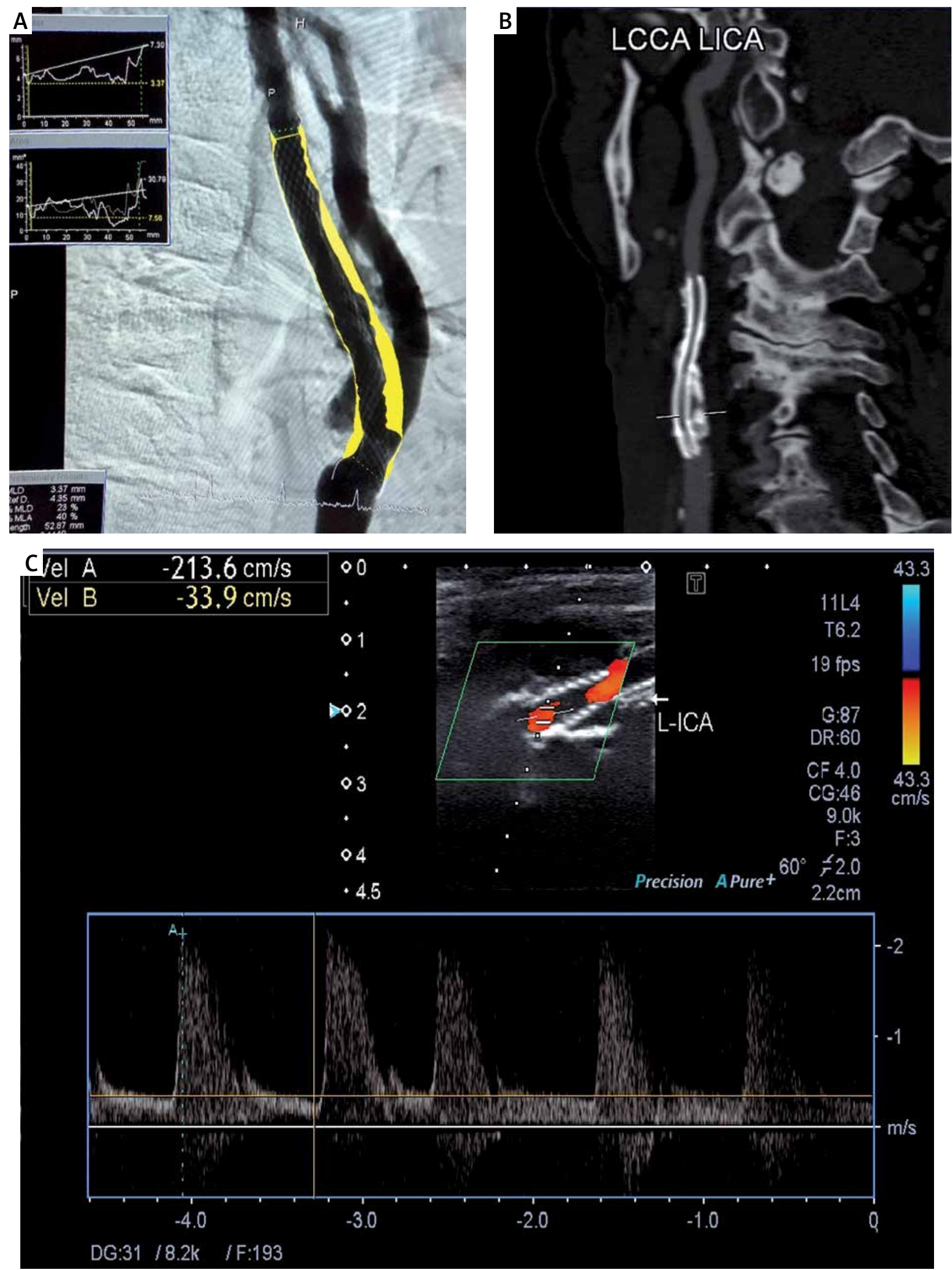

Figure 2. A - QCA assessment after stent implantation and postdilation. B - Control CT scan visualization of stent apposition in LICA stenosis. C - Postprocedural Doppler ultrasound revealing suboptimal result of CAS procedure 
US). Final angiography demonstrated good apposition of the stent and confirmed patency of the left carotid artery (Figure $2 \mathrm{~A}$ ). On the $5^{\text {th }}$ day after the procedure our patient presented new symptoms of left hemisphere transient ischemic attack (TIA) without any findings in the $\mathrm{CT}$ scan. Brain magnetic resonance imaging (MRI) scans revealed a few new microlesions in the left hemisphere. Due to transient neurological deterioration the patient was examined by a neurologist and standard pharmacological treatment was recommended. After the next 3 days the diagnosis of COVID-19 infection was confirmed and the patient had to stay at the hospital for the next 10 days. We observed temporary weakness and elevation of inflammatory biomarkers without any respiratory dysfunction. Finally after recovering from COVID-19 infection all ischemic symptoms reversed; the postprocedural Doppler ultrasound and CT-angio scans revealed a patent and properly apposed stent in the left carotid artery with $3.0 \mathrm{~mm}$ minimal lumen diameter (Figures $2 \mathrm{~B}, \mathrm{C}$ ).

Based on the presented, very challenging case we can assume that lithoplasty technique might be an effective and safe tool for the treatment of calcified carotid lesions. We need consecutive cases to confirm clinical usefulness of the IVL technique in this vascular bed. It is worth mentioning that intravascular lithotripsy seems to be very safe and well tolerated while dilating the carotids. Our first experiences after peripheral IVL S4 catheter usage are encouraging. The extended working segment of the S4 catheter with five active emitters could be helpful and accurate when treating long, calcified lesions in different vascular regions. Intravascular ultrasound (IVUS) and optical coherence tomography (OCT) could be adjunctive tools to identify calcium deposits and optimize the final results of stenting procedures.

\section{Acknowledgments}

We would like to thank Mr. Zbigniew Jakubas for his financial support in implementation of the project.

\section{References}

1. Auberson D, Gray WA. A new Sheriff in town. Vascular calcium meets its match. Catheter Cardiovasc Interv 2019; 93: 343-4.

2. Fanelli F, Cannavale A, Gazzetti M, et al. Calcium burden assessment and impact on drug-eluting ballonons in peripheral arterial disease. Cardiovasc Intervent Radiol 2014; 37: 898-907.

3. Dini CS, Tomberli B, Mattesini A, et al. Intravascular lithotripsy for calcific coronary and peripheral artery stenoses. Eurointervention 2019; 15: 714-21.

4. Brinton T, Hill J, Ali Z, et al. DISRUPT CAD: a multicenter, prospective, single-arm study of percutaneous lithoplasty prior to stent implantation in heavily calcified coronary lesion. J Am Coll Cardiol 2016; 68 TCT Suppl.

5. Brodmann M, Werner M, Holden A, et al. Primary outcomes and mechanism of action of intravascular lithotripsy in calicified, femoropoliteal lesions: results of DISRUPT PAD II. Catheter Cardiovasc Interv 2019; 93: 335-42.
6. Vadala G, Galassi AR, Nerla R, et al. Shockwave intravascular lithoplasty for the treatment of calcified carotid artery stenosis: a very early single-center experience. Catheter Cardiovasc Interv 2020; 96: E608-13.

7. Grillo P, Tripolino C, Tassone EJ, et al. Critical calcified carotid stenosis treated with Shockwave lithoplasty. Arch Med Sci Atherocler Dis 2018; 3: e164-5. 